\title{
RAMIFICATION OF KLEIN COVERINGS
}

\author{
CABIRIA ANDREIAN CAZACU
}

The aim of this paper is to extend the Hurwitz formula on the ramification of the covering surfaces [7], [8], as well as its generalizations by S. Stoilow [11], [12], ourselves [3] and I. Bârză [6] from Riemann coverings to Klein coverings. In what follows we shall use definitions and notations due to N. L. Alling and N. Greenleaf [2].

1. Let $\mathscr{X}=(X, \mathscr{A})$ and $\mathscr{Y}=(Y, \mathscr{B})$ be connected Klein surfaces, orientable or non-orientable, with or without border: $X$ and $Y$ will be connected two-manifolds with countable bases (surfaces) $\mathscr{A}=\{(U, z)\}$ and $\mathscr{B}=\{(W, w)\}$ dianalytic atlasses, $B_{X}$ and $B_{Y}$ the border (boundary) of $X$ and $Y$, respectively, [2], Section 2.

A morphism of Klein surfaces $T: \mathscr{X} \rightarrow \mathscr{Y}$ is a continuous mapping $T: X \rightarrow Y$, with the properties that $T\left(B_{X}\right) \subset B_{Y}$ and that for all points $P \in X$ there exist dianalytic charts $(U, z) \in \mathscr{A}$ and $(W, w) \in \mathscr{B}$ about $P$ and $p=T(P)$, respectively, and an analytic function $F$ on $z(U) \subset C^{+}=\{z \in C: y \geqq 0\}$, such that $T \mid U=w^{-1} \circ \varphi \circ F \circ z$. Here $\varphi: C \rightarrow C^{+}$is the folding mapping $\varphi(z)=x+i|y|$ that folds $\boldsymbol{C}$ over $\boldsymbol{C}^{+}$, [2], Section 4.

Evidently, if $B_{Y}=\emptyset$, it follows $B_{X}=\emptyset$ and one may give up $\varphi$, but if $B_{Y} \neq \emptyset$, even for Riemann surfaces, i.e., for $X$ and $Y$ orientable surfaces, this concept of morphism differs from the classical one ([9], I, II) since it permits the folding over $B_{Y}$. Starting from Stoilow's topological theory of Riemann surfaces [12], it was natural to compare this concept with that of the interior transformation (continuous, open and 0-dimensional (light) mapping) and we proved [5]:

Theorem 1. Non-constant morphisms of Klein surfaces are topologically equivalent to interior transformations in the sense of Stoilow.

It is obvious that a non-constant morphism is an interior transformation, but Stoilow's methods extend, so that we generalized his local inversion theorem for interior mappings $T: X \rightarrow Y$ between surfaces $X$ and $Y$ as above. Namely, if $P \in$ $X \backslash T^{-1}\left(B_{Y}\right)$, then in the neighbourhood of $P$ the map $T$ is topologically equivalent to $w=z^{h}$, and if $P \in T^{-1}\left(B_{Y}\right)$, to $w=\varphi \circ z^{h}$, which corresponds to the local normal form of a morphism ([2], p. 30). If $T: X \rightarrow Y$ is an interior map, and if we organize $Y$ with a dianalytic structure as a Klein surface $\mathscr{Y}$, this structure is lifted by means of $T$ in a unique way to $X$ yielding a Klein surface $\mathscr{X}$ such that $T$ becomes a morphism $\mathscr{X} \rightarrow \mathscr{Y}$. 
On the model of Stoilow's definition of Riemann covering ([12], Chapter II, I, 2 and Chapter V, III, 4) we call each triple, $(X, T, Y)$ where $T: X \rightarrow Y$ is an interior transformation between the surfaces $X$ and $Y$ a Klein covering.

The problem of the ramification of a Klein covering being of topological nature we shall not further refer to the Klein structure of our surfaces $X$ and $Y$, which we always suppose to be connected and of finite Euler characteristic $\varrho_{X}$ and $\varrho_{Y}$, respectively.

2. Let us first shortly resume the previous results: the Hurwitz formula and its generalizations in the case of the coverings without folds, i.e., $T^{-1}\left(B_{Y}\right)=B_{X}$, in particular in the case $B_{Y}=\emptyset$.

1) Hurwitz formula ([7], p. 54) is proved in Kerékjártó's book ([8], p. 158-159) for both orientable and non-orientable, compact surfaces with or without border $X$ and $Y$ under the hypothesis $T^{-1}\left(B_{Y}\right)=B_{X}$. The ramification number $r$ of the relatively unbordered $n$-sheeted covering $T: X \rightarrow Y$ satisfies the relation

$$
r=\varrho_{X}-n \varrho_{Y} .
$$

(See also Ahlfors' generalization of this formula [1], p. 168, and [10], p. 324.)

In Stoilow's theory $X$ and $Y$ are orientable surfaces without border and the Hurwitz formula holds for the total covering, which is realized by any interior mapping $T: X \rightarrow Y$ with the following property: for each infinite sequence of points $P_{v} \in X$ which tends to the ideal boundary $\partial X$ of $X$ (i.e., has no accumulation point in $X$, notation: $\left.P_{v} \rightarrow \partial X\right)$, its projection $p_{v}=T\left(P_{v}\right)$ tends to $\partial Y$. This is in particular the case when $X$ and $Y$ are compact ([12], Chapter VI, II-III).

2) In 1933, [11], Stoilow extended formula (1) by introducing the partially regular covering $T: X \rightarrow Y$, characterized by the existence of a finite family of mutually disjoint Jordan curves $\gamma$ on $Y$ with the following properties: $\left(\beta_{1}\right)$ for each sequence $P_{v} \rightarrow \partial X$, its projection $p_{v} \rightarrow \gamma \cup \partial Y$ (i.e., $p_{v}$ has accumulation points only on $\gamma$ ), and $\left(\beta_{2}\right)$ the set $T^{-1}(\gamma)$ is either compact or empty. Then the family $\gamma$ decomposes $Y$ into a finite number of regions $Y_{i}$ of finite characteristic $\varrho_{i}$ and totally covered with $n_{i}$ sheets by $T^{-1}\left(Y_{i}\right)$. For such a partially regular covering Stoilow obtained the formula ([11], [12], Chapter VI, IV)

$$
r=\varrho_{X}-\sum n_{i} \varrho_{i} .
$$

3) In 1960, [3], we considered - again in the orientable, unbordered case - a family $\gamma$ on $Y$, consisting of a finite number of mutually disjoint Jordan curves and of a finite number of Jordan arcs with the end points in well-determined points on $Y$, which we called knots, or elements of $\partial Y$. An arc of $\gamma$ can meet another arc or curve of $\gamma$ only in a knot. The set of knots will be denoted by $\mathscr{N}$.

We supposed that the interior transformation $T: X \rightarrow Y$ satisfies the condition $\left(\beta_{1}\right)$ but we gave up the condition $\left(\beta_{2}\right)$ and introduced the local condition $\left(L \beta_{2}\right)$ : a point $p \in \gamma$ satisfies $\left(L \beta_{2}\right)$ if there exists a neighbourhood $v$ of $p$ such that the preimage of the component of $v \cap \gamma$, which contains $p$, is either relatively compact in $X$ 
or empty. In this way it was natural to define the exceptional points $p \in \gamma$ which do not satisfy $\left(L \beta_{2}\right)$, and we proved that the set $\mathscr{E}$ of these points is finite or empty.

The surface $Y$ is again decomposed by $\gamma$ into a finite number of regions $Y_{i}$ of finite characteristic $\varrho_{i}$ and totally covered by $n_{i}$ sheets.

We chose a family $\gamma^{\prime}$ of mutually disjoint Jordan curves from $\gamma$, which contains all Jordan curves of $\left.\gamma \backslash(\mathscr{E} \cup \mathscr{N})^{*}\right)$. Let $\mathscr{E}_{\gamma^{\prime}}$ be the set of exceptional points of $\gamma^{\prime}$ with respect to itself (i.e., the set of the points $p \in \gamma^{\prime}$ for which $T^{-1}\left(v \cap \gamma^{\prime}\right)$ is not relatively compact in $X$ for every neighbourhood $v$ of $p$ ). The curves $\gamma^{\prime}$ with $\mathscr{E}_{\gamma^{\prime}} \neq \emptyset$ are decomposed by $\mathscr{E}_{\gamma^{\prime}}$ into open Jordan arcs and similarly $\left(\gamma \backslash \gamma^{\prime}\right) \backslash(\mathscr{E} \cup \mathscr{N})$ consists of open Jordan arcs. We called these arcs cross cuts and denoted them by $\gamma_{j}^{\prime \prime}$, the family contained in $\gamma^{\prime}$ by $\left\{\gamma_{j}^{\prime \prime}\right\}^{1}$ and the rest by $\left\{\gamma_{j}^{\prime \prime}\right\}^{2}$. Every cross cut $\gamma_{j}^{\prime \prime}$ is covered by $s_{j}$ sheets.

Further, we denoted by $v(p)$ the number of sheets of the covering over a point $\left.p \in Y^{* *}\right)$, designated by $p_{k}$ the points of the set $\mathscr{E}_{\gamma^{\prime}} \cup\left(\mathscr{E} \cup \mathcal{N} \backslash \gamma^{\prime}\right)$ and wrote $v\left(p_{k}\right)=v_{k}$.

Then we obtained the following generalization of the Hurwitz-Stoilow formula (2):

$$
r=\varrho_{X}-\sum n_{i} \varrho_{i}-\sum s_{j}+\sum v_{k} .
$$

Its importance is due to its wide application possibilities, for instance to the regions of the exhaustion of a Riemann surface and thus to the study of the ramification of Riemann coverings in general [4].

4) The formulae (2) and (3) remain valid for non-orientable surfaces without border, as it was proved by I. Bârză [6].

3. We shall now consider the general case of a Klein covering $T: X \rightarrow Y$ and obtain in Theorem 2 the formula (4), which, assuming the particular hypotheses presented above, reduces to the results 1)-4). It thus remains to concentrate on the case $T^{-1}\left(B_{Y}\right) \neq B_{X}, X$ and $Y$ being orientable or non-orientable, $B_{Y} \neq \emptyset$ but $B_{X}$ being empty or not. It should be mentioned that even in the case $T^{-1}\left(B_{Y}\right)=B_{X}$ formula (4) will bring new information since the assertions 2) -4) have been established only for $B_{Y}=\emptyset$ but according to (4) they are also true if $B_{Y} \neq \emptyset$.

3.1. Since $X$ and $Y$ have finite characteristics, we can represent them by means of homeomorphisms which do not influence the ramification of the Klein covering $T: X \rightarrow Y$ as subsets of compact surfaces $X$ and $Y$ with the same genus as $X$ and $Y$. Here $\boldsymbol{X}$ is orientable if and only if $X$ is orientable; the same holds for $\boldsymbol{Y}$ and $Y$. Under these homeomorphisms the ideal boundaries of $X$ and $Y$ correspond to a finite set of points $F_{X} \subset x$ and $F_{Y} \subset y$ and their borders to a finite family of mutually disjoint Jordan curves and $\operatorname{arcs} B_{X}$ on $\boldsymbol{X}$ and $B_{Y}$ on $\boldsymbol{Y}$, the arcs ending in points of $F_{X}$ and $F_{Y}$, respectively. For $X$ we shall write $B_{X}=B_{X}^{1} \cup B_{X}^{2}$ and $F_{X}=F_{X}^{1} \cup F_{X}^{2}$, where $B_{X}^{1}$ contains the Jordan curves of $B_{X}$ and $B_{X}^{2}$ its Jordan arcs,

*) We denote by $\gamma$ the family of curves and arcs, one curve or arc of the family as well as the set of the points of these curves and arcs; the same remark holds about $\gamma^{\prime}$.

**) $v(p)$ is the number of points in $T^{-1}(p)$ counted with their multiplicities. 
$F_{X}^{1}$ the isolated points of $B_{X} \cup F_{X}$ and $F_{X}^{2}$ the end points of the arcs from $B_{X}^{2}$. Moreover - without influencing the ramification of the covering - we can suppose ([5]) that $B_{X}^{2} \cup F_{X}^{2}$ consists of a finite number of mutually disjoint Jordan curves on $\boldsymbol{X}$, each point of $F_{X}^{2}$ giving exactly two end points of arcs of $B_{X}^{2}$. Similar notations will be used for $Y$.

3.2. On $Y$ we shall consider a family $\gamma$ like that of 3) but we let arcs from $\gamma$ have end points on $B_{Y} \cup F_{Y}$. The points of $\gamma \cap B_{Y}$ will also be called knots and the set of all the knots will again be designated by $\mathscr{N}$. The covering $(X, T, Y)$ will satisfy the condition $\left(\beta_{1}\right)$ for $\gamma \cup B_{Y} \cup F_{Y}$. Further, $\mathscr{E}$ will designate the set of the exceptional points of $\gamma$. Obviously $\mathscr{E} \cap B_{Y} \subset \mathscr{N}$. Besides $\mathscr{E}$ the covering can present exceptional points on $B_{Y}$ relatively to $B_{Y}$ itself. For such a point $p \in B_{Y}$ the pre-image $T^{-1}\left(v \cap B_{Y}\right)$ is not relatively compact for any neighbourhood $v$ of $p$. The set of these points will be denoted by $E$.

3.3. Another important set will be $\mathscr{R}$ - the set of the projections of the ramification points of the covering $T: X \rightarrow Y$.

The new type of coverings we are now considering may be characterized by one of the following equivalent conditions:

(i) $T^{-1}\left(B_{Y}\right) \neq B_{X}$, and

(ii) the covering presents folds over $B_{Y}$.

If $\mathscr{R} \cap B_{Y} \neq \emptyset$, then these conditions are fulfilled.

Before writing formula (4) we shall discuss in 3.4 and 3.5 the two new aspects that occur for the covering.

3.4. The case $\mathscr{R} \cap B_{Y} \neq \emptyset$. Let $p$ be a point of $B_{Y}$. Its pre-image $T^{-1}(p)$ consists of points $Q_{j} \in \dot{X}=X \backslash B_{X}, j=1, \ldots, \dot{\mathfrak{i}}(p)$, with the multiplicity $h_{j}$ (i.e., where locally $T$ is topologically equivalent to the mapping $w=\varphi \circ z^{h_{j}}$ ), and of points $P_{j} \in B_{X}$, $j=1, \ldots, \mathfrak{b}(p)$, with the multiplicity $k_{j}$ (i.e., where locally $T$ is topologically equivalent to one of the mappings $w=\varphi \circ z^{k_{j}}$ or $\left.w=\varphi \circ\left(-z^{k_{j}}\right)\right)$.

Let $v$ be a sufficiently small open neighbourhood of $p$ and $l, l^{*}$ the two open Jordan arcs in which $p$ divides the component of $v \cap B_{Y}$ that contains it. For each $Q_{j}$ the corresponding component of $T^{-1}(v)$ is a normal region (in Stoilow's sense [12], Chapter V, II) which covers $v$ under $T$ with $2 h_{j}$ sheets and $l \cup l^{*}$ with $h_{j}$ folds. Similarly, for each $P_{j}$ the corresponding component of $T^{-1}(v)$ is a normal region and covers $v$ under $T$ with $k_{j}$ sheets and $l \cup l^{*}$ with $\left(k_{j}-1\right)$ folds and 2 borders. More precisely, if $k_{j}$ is odd, each of the arcs $l$ and $l^{*}$ will be covered by $\left(k_{j}-1\right) / 2$ folds and a border, and if $k_{j}$ is even, one of them will be covered by $k_{j} / 2$ folds and the other by $\left(k_{j}-2\right) / 2$ folds and 2 borders.

In order to preserve the form (1) of the Hurwitz formula for total coverings, we shall define the ramification order of a point $Q_{j}$ as usual by $\left(h_{j}-1\right)$ but the ramification order of a point $P_{j}$ by $\left.\left(k_{j}-1\right) / 2,{ }^{*}\right)$ so that the ramification of the covering at

*) This definition has also an interpretation in connection with the double coverings. 
$p$ will be

$$
r(p)=\sum_{j=1}^{i(p)}\left(h_{j}-1\right)+\frac{1}{2} \sum_{j=1}^{\mathfrak{b}(p)}\left(k_{j}-1\right) .
$$

A special role will be played in what follows by $\mathscr{R}_{1 / 2}=\left\{p \in \mathscr{R} \cap B_{Y}: h_{j}=1\right.$ for each $Q_{j} \in T^{-1}(p) \cap \stackrel{\circ}{X}$ and $k_{j}=1$ or 2 for each $P_{j} \in T^{-1}(p) \cap B_{X}$, with at least one $\left.k_{j}=2\right\}$. If $p \in \mathscr{R}_{1 / 2}$, then the number of the points $P_{j}$ with $k_{j}=2$ in $T^{-1}(p) \cap B_{X}$ will be $2 r(p)$. Thus $2 r(p)$ represents also the number of the folds that cover one of the $\operatorname{arcs} l$ or $l^{*}$ but transform at $p$ into two borders covering the arc $l^{*}$ or $l$, respectively.

At a point $p \in \stackrel{\circ}{Y}=Y \backslash B_{Y}$ the ramification will be taken as usual

$$
r(p)=\sum_{j=1}^{\mathbf{i}(p)}\left(h_{j}-1\right),
$$

where $T^{-1}(p)=\left\{Q_{1}, \ldots, Q_{\mathfrak{i}(p)}\right\} \subset \stackrel{\circ}{X}$ and $Q_{j}$ has the multiplicity $h_{j}$ (i.e., locally $T$ is topologically equivalent to $\left.w=z^{h_{j}}\right)$ and the ramification order $\left(h_{j}-1\right)$.

Evidently, $p \in \mathscr{R}$ if and only if $r(p)>0$ and the ramification number of the covering $r=\sum_{p \in \mathscr{R}} r(p)$.

3.5. Folds "ending at a point" of $E \cup F_{Y}^{2}$. If $p \in E$, there are folds or borders (at least one) which cover one of the arcs $l$ or $l^{*}$ without covering $p$. Let $f(p)$ and $b(p)$, respectively, be the numbers of these folds and borders. Such a fold (border) represents an asymptotic way in ${ }_{X}^{\circ}$ (on $B_{X}$ ) for $T$ with the asymptotic value $p$ and will be called fold or border "ending at $p$ ".

The same notations $f(p)$ and $b(p)$ will be introduced for the points $p \in F_{Y}^{2}$. (If we do not suppose, as indicated in 3.1, that $B_{Y}^{2} \cup F_{Y}^{2}$ consists of Jordan curves and in a sufficiently small neighbourhood $v$ of $p$ one has exactly two arcs $l$ and $l^{*}$ of $B_{Y}^{2}$ ending at $p$, then $p$ can be an end point for more than two arcs like $l$ or for a single one, and $f(p)$ as well as $b(p)$ will be the numbers of folds or borders "ending at $p$ " over all these arcs. The sum $\sum_{p \in F_{Y}^{2}} f(p)$ is independent of this [5].)

3.6. With these remarks and notations we can formulate

Theorem 2. Let $T: X \rightarrow Y$ be a Klein covering, $\gamma$ a family of curves and arcs as in 3) but which, if $B_{Y} \neq \emptyset$, may have end points on $B_{Y}$, too, and suppose that $T$ satisfies the condition $\left(\beta_{1}\right)$ with respect to $\gamma \cup B_{Y} \cup F_{Y}$ (if $P_{v} \rightarrow B_{X} \cup F_{X}$, then $p_{v} \rightarrow \gamma \cup B_{Y} \cup F_{Y}$ ). The family $\gamma$ decomposes $Y$ into the regions $Y_{i}$ with the characteristics $\varrho_{i}$ and number of the sheets $n_{i}: Y \backslash\left(\gamma \cup B_{Y}\right)=\cup Y_{i}$. We choose a family $\gamma^{\prime}$ of mutually disjoint Jordan curves from $\gamma$, so that it contains all those curves from $\gamma$ without exceptional points with respect to themselves (i.e., if a curve $\gamma$ has $\mathscr{E}_{\gamma}=\emptyset$, then it will be included in the family $\left.\left.\gamma^{\prime}\right) .^{*}\right)$ As in 3) we determine the cross cuts $\gamma_{j}^{\prime \prime}$ covered by $s_{j}$ sheets. Further, let $p_{k}$ be the points of the set $\mathscr{E}_{\gamma^{\prime}} \cup\left[(\mathscr{E} \cup \mathscr{N}) \backslash\left(\gamma^{\prime} \cup B_{Y}\right)\right]$ and $q_{l}$ the points of $E \cup F_{Y}^{2}, v_{k}=v\left(p_{k}\right)$

*) There is a difference between the actual construction of $\gamma^{\prime}$ and that of 3) but this does not influence the result. 
and $f_{l}=f\left(q_{l}\right)$. Then the ramification number of the covering is given by the formula

$$
r=\varrho_{X}-\sum n_{i} \varrho_{i}-\sum s_{j}+\sum v_{k}-\frac{1}{2} \sum f_{l} .
$$

Remark 1. We can admit that $\gamma$ contains, besides curves and arcs as before, a finite number of points $p \in \dot{Y}^{\circ}$ such that for each neighbourhood $v$, its pre-image $T^{-1}(v)$ is not relatively compact in $X$. These points will be interpreted as curves $\gamma^{\prime}$ reduced to a point and be considered in $\mathscr{E}_{\gamma^{\prime}}$, hence denoted by $p_{k}$. Formula (4) holds in this case too, with the mention that the sum $\Sigma v_{k}$ contains also the terms corresponding to these points $p_{k}$.

Remark 2. Evidently (4) refers also to the case of the total covering with folds: $\gamma=\emptyset$. If $f(p)=0$ for each $p \in B_{Y} \cup F_{Y}^{2}$, then (4) takes the form (1). This case may also be proved directly by counting the simplexes of the covering as for the classical Hurwitz formula ([10], p. 324). This was done in [2, p. 43], for the case of the unramified double covering of a compact Klein surface.

The proof of (4) will be given in the next two sections, 4. and 5., by adapting the method we used in [3] in order to prove (3). Ahlfors' formula for the addition of the characteristics will remain the main tool.

The finiteness of the sets $\mathscr{E}, E, \mathscr{R}$ as well as the total covering and the finite numbers of sheets $n_{i}, s_{j}, v_{k}$ over each $Y_{i}, \gamma_{j}^{\prime \prime}, p_{k}$, respectively, and the finiteness of the numbers $f_{l}$ are proved by similar devices as in [3]. (For details see [5].)

4. Let us first prove Theorem 2 in the special case $\mathscr{R} \cap\left(\gamma \cup B_{Y}\right)=\mathscr{R}_{1 / 2}$ and $\mathscr{E} \cup \mathscr{N} \subset \gamma^{\prime} \cup B_{Y}$.

Besides the cross cuts $\left\{\gamma_{j}^{\prime \prime}\right\}$, we shall also consider a family of cross cuts $\left\{B_{m}^{\prime \prime}\right\}$ on $B_{Y}$, namely, the family of Jordan arcs which appear on $B_{Y}$ when one takes out the points of $\mathscr{R}_{1 / 2} \cup E$. These arcs have their end points in the set $M=\mathscr{R}_{1 / 2} \cup E \cup F_{Y}^{2}$ and each $B_{m}^{\prime \prime}$ is covered by $\sigma_{m}$ sheets.

We denote by $X_{u}$ and $X_{u i}^{v}$ the components of $X \backslash T^{-1}\left(\gamma^{\prime} \cup B_{Y}\right)$ and $X \backslash T^{-1}\left(\gamma \cup B_{Y}\right)$, respectively, where $X_{u i}^{v}$ are the components of $X_{u} \cap T^{-1}\left(Y_{i}\right)$, and remark that $\left(X_{u i}^{v}, T \mid, Y_{i}\right)$ is a total covering in Stoilow's sense, so that we can apply the Hurwitz formula (1) to get

$$
r_{u i}^{v}=\varrho_{u i}^{v}-n_{u i}^{v} \varrho_{i},
$$

where $r_{u i}^{v}$ is the ramification of the covering, $n_{u i}^{v}$ the number of sheets and $\varrho_{u i}^{v}$ the characteristic of $X_{u i}^{v}$.

If $\varrho_{u}$ is the characteristic of $X_{u}$ and $N_{1}$ or $N_{B}$, respectively, represents the number of cross cuts in the decomposition $X \backslash T^{-1}\left(\gamma^{\prime} \cup B_{Y}\right)=\cup X_{u}$ which comes from $T^{-1}\left(\gamma^{\prime}\right)$ and $T^{-1}\left(B_{Y}\right)$, respectively (we remark that $\gamma^{\prime} \cap B_{Y}=\emptyset$ ), then by Ahlfors' formula

$$
\varrho_{X}=\sum \varrho_{u}+N_{1}+N_{B}
$$


Further, let $N_{2}$ be the number of cross cuts which appear when we continue the decomposition: $\cup X_{u} \backslash T^{-1}\left(\left\{\gamma_{j}^{\prime \prime}\right\}^{2}\right)=\cup X_{u i}^{v}$. The same formula gives

$$
\sum \varrho_{u}=\sum \varrho_{u i}^{v}+N_{2} .
$$

By repeating the device used in [3], we obtain again

$$
N_{1}+N_{2}=\sum s_{j}-\sum v_{k}
$$

$\left(N_{1}=\sum^{1} s_{j}-\sum v_{k}, N_{2}=\sum^{2} s_{j}\right.$, where $\sum^{1}$ and $\sum^{2}$ extend to the families $\left\{\gamma_{j}^{\prime \prime}\right\}^{1}$ and $\left\{\gamma_{j}^{\prime \prime}\right\}^{2}$, respectively, and $\left.p_{k} \in \mathscr{E}_{\gamma^{\prime}}\right)$.

In order to evaluate $N_{B}$ we remark that a cross cut from $T^{-1}\left(\left\{B_{m}^{\prime \prime}\right\}\right)$ determines a fold and counted with its end points contributes with 2 to the sum $\sum_{p \in M}(2 r(p)+$ $f(p))$. Therefore

$$
N_{B}=\frac{1}{2} \sum_{p \in M}(2 r(p)+f(p)) \text {. }
$$

Since $r=\sum r_{u i}^{v}+\sum_{p \in M} r(p)$ and $\sum_{p \in M} f(p)=\sum f_{l}$, formula (4) follows from (5)-(9).

Remark 3. By the device used in the calculation of $N_{1}$, [3], one proves that

$$
2\left(\sum \sigma_{m}-\sum_{p \in M} v(p)\right)=\sum_{p \in M}(2 r(p)+f(p)+b(p)) .
$$

5. The general case. In order to obtain from the general Klein covering $T: X \rightarrow Y$ with the condition $\left(\beta_{1}\right)$ for a family $\gamma \cup B_{Y} \cup F_{Y}$ a covering from the special case 4, we suitably modify the method used in [3], 8. Namely, we introduce the sets $\mathscr{R}^{*}=$ $(\mathscr{R} \cap \gamma) \backslash(\mathscr{E} \cup \mathscr{N}), \mathscr{R}^{* *}=\left(\mathscr{R} \cap B_{Y}\right) \backslash \mathscr{R}_{1 / 2}$ and $A=\left(\mathscr{R}^{*} \cup \mathscr{E} \cup \mathscr{N}\right) \backslash B_{Y}$, and we choose a set of sufficiently small open neighbourhoods $v$ for the points $p \in A \cup \mathscr{R}^{* *}$, such that the closed neighbourhoods $\bar{v}$ are mutually disjoint and the following conditions are fulfilled: For each $p \in A, \bar{v} \subset \grave{Y}, \bar{v}$ is a Jordan domain bounded by a Jordan curve $c$; $(\bar{v} \backslash\{p\}) \cap(\mathscr{E} \cup \mathscr{N} \cup \mathscr{R})=\emptyset$ and $\bar{v} \cap \gamma$ consists of a finite number of Jordan arcs with an end point at $p$ and another on $c$; these arcs decompose $v$ into sectors; any non-compact component of $T^{-1}(\bar{v})$ does not intersect $T^{-1}(p)$. For each $p \in \mathscr{R}^{* *}$, $\bar{v} \backslash B_{Y} \subset \stackrel{Y}{Y}, \bar{v} \cap B_{Y}$ is a Jordan arc $a p b$ while $\bar{v}$ is a Jordan domain bounded on $y$ by $a p b$ and a Jordan arc $c$ which is contained in $Y$ except for its end points $a$ and $b$; $(\bar{v} \backslash\{p\}) \cap(\mathscr{E} \cup \mathscr{N} \cup \mathscr{R} \cup E)=\emptyset$, and $\bar{v} \cap \gamma$ has the same properties as in the case $p \in A$; any non-compact component of $T^{-1}(\bar{v})$ does not intersect $T^{-1}(p)$ and every compact component contains a single point of $T^{-1}(p)$.

First we take out of $X$ the union of the non-compact components of $T^{-1}(\bar{v})$ for all $p \in A$ and obtain a surface $X^{*}$.

According to [3], 7, these components are either simply connected and separated from $X^{*}$ by a cross cut or doubly connected and separated from $X^{*}$ by a Jordan curve, such that we have

$$
\varrho_{X}=\varrho_{X^{*}} \quad \text { and } \quad r=r^{*} \text {, }
$$


where $\varrho_{X^{*}}$ means the characteristic of $X^{*}$ and $r^{*}$ the ramification number of the covering $\left(X^{*}, T \mid, Y\right)$.

Secondly, we take out of $X^{*}$ the non-compact components of $T^{-1}(\bar{v})$ and the relatively compact components of $T^{-1}(v)$ for all $p \in \mathscr{R}^{* *}$, obtaining a surface $\tilde{X}$ with the characteristic $\varrho_{\tilde{X}}$, and take out from $Y$ the neighbourhoods $v$ for all $p \in \mathscr{R}^{* *}$, obtaining the surface $\tilde{Y}$.

By a direct computation we prove that the non-compact components of $T^{-1}(\bar{v})$, $p \in \mathscr{R}^{* *}$, are again of the same type as described for $\left.p \in A .^{*}\right)$ If $T^{-1}(p)=$ $\left\{Q_{1}, \ldots, Q_{\mathrm{i}(p)}, P_{1}, \ldots, P_{\mathrm{b}(p)}\right\}, Q_{j} \in \dot{X}^{*}, P_{j} \in B_{X^{*}}$, as in 3.4 , the component of $T^{-1}(\bar{v})$ containing $Q_{j}$ will be a Jordan domain included in $\dot{X}^{*}$ and the component containing $P_{j}$ will be homeomorphic to a half disc separated from $\tilde{X}$ by a Jordan arc projected by $T$ on $c$. Therefore with Alhfors' formula we have

$$
\varrho_{\tilde{X}}=\varrho_{X}+\sum_{p \in \mathscr{R} * *} \mathfrak{i}(p) .
$$

On the other hand, taking out a relatively compact component of $T^{-1}(v)$ for $p \in \mathscr{R}^{* *}$ which contains a point $Q_{j}$ with the multiplicity $h_{j}$, we have $2 h_{j}$ new ramification points of order $1 / 2$ projected over $a$ and $b$. Similarly, if the component contains a point $P_{j}$ with the multiplicity $k_{j}$, we have $\left(k_{j}-1\right)$ new ramification points of order $1 / 2$ projected over $a$ and $\left.b .^{* *}\right)$ Therefore the ramification number $\tilde{r}$ of the covering $(\tilde{X}, T \mid, \widetilde{Y})$ will be given by the relation

$$
\tilde{r}=r+\sum_{p \in \mathscr{R}^{* *}} \mathfrak{i}(p) .
$$

However, the covering $(\tilde{X}, T \mid, \tilde{Y})$ satisfies the condition $\left(\beta_{1}\right)$ with respect to the family of curves and arcs $\tilde{\gamma}=\left(\gamma \backslash \bigcup_{p \in A \cup \mathscr{R} * *} v\right) \cup\left(\bigcup_{p \in A} c\right)$ and to $B_{\tilde{Y}} \cup F_{\tilde{Y}}$, the family $\tilde{\gamma}^{\prime}$ consisting of the curves $c$ for $p \in A$ and the former curves $\gamma^{\prime}$ which do not intersect $A$. Let us remark that $\mathscr{E}_{c}=\emptyset$ for each $p \in A$, hence $\mathscr{E}_{\tilde{\gamma}^{\prime}}=\emptyset$. Further, $B_{\tilde{Y}}$ is obtained from $B_{Y}$ replacing the arc $a p b$ by the corresponding arc $c$ for each $p \in \mathscr{R}^{* *}$. It follows that $F_{\bar{Y}}^{2}=F_{Y}^{2}$. We denote by $\tilde{E}$ the set of the points of $B_{\tilde{Y}}$ which are exceptional with respect to $B_{\tilde{Y}}$. Obviously $\widetilde{E}$ is obtained from $E$ replacing each point $p \in \mathscr{R}^{* *} \cap E$ by the corresponding pair $\{a, b\}$.

In order to apply (4) to the covering $(\tilde{X}, T \mid, \tilde{Y})$, let us denote by $\tilde{Y}_{\lambda}$ the components of $\tilde{Y} \backslash\left(\tilde{\gamma} \cup B_{\tilde{\gamma}}\right.$ ), by $\tilde{\gamma}_{\mu}$ the cross cuts (of the type $\left.\left\{\gamma_{j}^{\prime \prime}\right\}^{2}\right)$ determined on $\tilde{\gamma} \backslash \tilde{\gamma}^{\prime}$, by $\tilde{\varrho}_{\lambda}$ the characteristic of $\tilde{Y}_{\lambda}$, by $\tilde{n}_{\lambda}$ and $\tilde{s}_{\mu}$ the number of sheets over $\tilde{Y}_{\lambda}$ and $\tilde{\gamma}_{\mu}$, by $\tilde{q}$ a point of $\tilde{E} \cup F_{\tilde{Y}}^{2}$ and by $\tilde{f}(\tilde{q})$ the number of folds of $(\tilde{X}, T \mid, \tilde{Y})$ "ending at $\tilde{q}$ ". In this

*) Let $\mathscr{V}$ be the interior of such a component. The covering $(\mathscr{V}, T \mid, v)$ satisfies $\left(\beta_{1}\right)$ with respect to $(\gamma \cap v) \cup \partial v$ and $v$ is decomposed by the arcs from $\gamma \cap v$ into sectors. The number of the sheets over a sector is at least equal to the number of the sheets over each arc from $\gamma \cap v$ on its boundary and at least twice the number of the folds over one of the arcs $p a$ and $p b$ on its boundary. The preimages of these arcs and of $p a$ and $p b$ give cross cuts of $\mathscr{V}$. The assertion follows using Ahlfors' formula for the characteristic of $\mathscr{V}$ and the Hurwitz formula (1) for each covering of the sectors.

**) One sees why it was necessary to consider $\mathscr{R}_{1 / 2}$ in Section 4 . 
way formula (4) gives

$$
\tilde{r}=\varrho_{\tilde{X}}-\sum \tilde{n}_{\lambda} \tilde{\varrho}_{\lambda}-\sum \tilde{s}_{\mu}-\frac{1}{2} \sum \tilde{f}(\tilde{q}) .
$$

It remains to express (13) in terms of the covering $(X, T, Y)$.

One sees immediately that

$$
\sum \tilde{f}(\tilde{q})=\sum f_{l} .
$$

Indeed, if $q_{l} \in\left(E \cup F_{Y}^{2}\right) \backslash \mathscr{R}^{* *}$, then it is a point $\tilde{q}$ with $\tilde{f}(\tilde{q})=f_{l}$, and if $q_{l}=p \in \mathscr{R}^{* *}$, then it is replaced in $\tilde{E} \cup F_{\tilde{Y}}^{2}$ by the corresponding pair $\{a, b\}$ and $f_{l}=\tilde{f}(a)+\tilde{f}(b)$.

A region $\tilde{Y}_{\lambda}$ is either a neighbourhood $v$ for a point $p \in A$ and in this case $\tilde{\varrho}_{\lambda}=-1$ while $\tilde{n}_{\lambda}=v(p)$, or it is included in a uniquely determined region $Y_{i}$. Then $\tilde{\varrho}_{\lambda}=\varrho_{i}$, $\tilde{n}_{\lambda}=n_{i}$ and there exists a bijection between the regions $\tilde{Y}_{\lambda}$ of this last type and the regions $Y_{i},[3], 8$. Consequently

$$
\sum \tilde{n}_{\lambda} \tilde{\varrho}_{\lambda}=\sum n_{i} \varrho_{i}-\sum_{p \in A} v(p) .
$$

The curves $\gamma^{\prime}$ which do not intersect $A$ have no contribution to $\sum \tilde{s}_{\mu}$ nor to $\sum s_{j}$. Let $\gamma_{1}^{\prime}$ be the family of curves $\gamma^{\prime}$ with $\mathscr{E}_{\gamma^{\prime}}=\emptyset$ but which intersect $A$. Write $A_{1}=$ $A \cap \gamma_{1}^{\prime}$. The curves $\gamma_{1}^{\prime}$ have no contribution to $\sum s_{j}$ but yield $\sum_{p \in A_{1}} v(p)$ in $\sum \tilde{s}_{\mu}$. The other curves $\gamma^{\prime}$ with $\mathscr{E}_{\gamma^{\prime}} \neq \emptyset$ decompose into cross cuts of the family $\left\{\gamma_{j}^{\prime \prime}\right\}^{1}$ and these cross cuts as well as those from the family $\left\{\gamma_{j}^{\prime \prime}\right\}^{2}$ yield cross cuts $\tilde{\gamma}_{\mu}$. Namely, if a cross cut $\gamma_{j}^{\prime \prime}$ contains the points $p \in A$, then $\gamma_{j}^{\prime \prime}$ contributes with $s_{j}+\sum v(p)$ to $\sum \tilde{s}_{\mu}$. Hence

$$
\sum \tilde{s}_{\mu}=\sum s_{j}+\sum \sum_{p \in A_{1} \cup A_{2}} v(p),
$$

where $A_{2}=A \cap \gamma^{\prime \prime}$ and $\gamma^{\prime \prime}$ is the family of all the cross cuts $\gamma_{j}^{\prime \prime}$.

Therefore (11)-(16) imply

$$
r=\varrho_{X}-\sum n_{i} \varrho_{i}-\sum s_{j}+\sum \sum \in A \backslash\left(A_{1} \cup A_{2}\right) v(p)-\frac{1}{2} \sum f_{l}
$$

and it is easy to verify that $A \backslash\left(A_{1} \cup A_{2}\right)=\left\{\mathscr{E}_{\gamma^{\prime}} \cup\left[(\mathscr{E} \cup \mathscr{N}) \backslash \gamma^{\prime}\right]\right\} \backslash B_{Y}$, so that $\sum v(p)$ in (17) is equal to $\sum v_{k}$ in (4).

6. Finally, let us establish Theorem 2 in the case mentioned in Remark 1 in 3.4 when $\gamma$ also contains a finite number $M$ of points of $\stackrel{\circ}{Y}$. As indicated in 3.4, we consider these points in $\mathscr{E}_{\gamma^{\prime}}$, denote them by $p_{k}$ and suppose (if necessary by a change of numeration) that they correspond to the indices $k=1, \ldots, M$. Set for each of these $k, T^{-1}\left(p_{k}\right)=\left\{Q_{k 1}, \ldots, Q_{k \mathfrak{i}_{k}}\right\} \subset \stackrel{\circ}{X}$, every point $Q_{k j}$ having the multiplicity $h_{k j}, \hat{X}=X \backslash \bigcup_{k=1}^{M} T^{-1}\left(p_{k}\right)$ and $\hat{Y}=Y \backslash\left\{p_{1}, \ldots, p_{M}\right\}$.

We can apply formula (4) to the covering $(\hat{X}, T \mid, \hat{Y})$ so that the corresponding ramification number $\hat{r}$ is given by

$$
\hat{r}=\varrho_{X}-\sum n_{i} \varrho_{i}-\sum s_{j}+\sum_{k>M} v_{k}-\frac{1}{2} \sum f_{l},
$$


where $\varrho_{\hat{X}}$ is the characteristic of $\hat{X}$ and $Y_{i}$ are the components of $Y \backslash\left(\gamma \cup B_{Y}\right)$, the points $p_{1}, \ldots, p_{M}$ being included in $\gamma$.

On the other hand,

$$
r=\hat{r}+\sum_{k=1}^{M}\left(\sum_{j=1}^{\mathfrak{i}_{k}}\left(h_{k j}-1\right)\right)=\hat{r}+\sum_{k=1}^{M} v_{k}-\sum_{k=1}^{M} \mathfrak{i}_{k}
$$

and $\varrho_{\bar{X}}=\varrho_{X}+\sum_{k=1}^{M} \mathfrak{i}_{k}$ so that the formula (4) is true for the covering $(X, T, Y)$, too.

\section{References}

[1] AhLFons, L.: Zur Theorie der Überlagerungsflächen. - Acta Math. 65, 1935, 157-194.

[2] Alling, N. L., and N. Greenleaf: Foundations of the theory of Klein surfaces. - Lecture Notes in Mathematics 219. Springer-Verlag, Berlin-Heidelberg-New York, 1971.

[3] Andreian Cazacu, C.: Über eine Formel von S. Stoilow. - Rev. Roumaine Math. Pures Appl. $5,1960,59-74$.

[4] Andreian Cazacu, C.: Clase de acoperiri riemanniene I, II. - An. Univ. Bucureşti, Şti. Natur. 13:2, 1964, 55-75; 14:1, 1965, 71-95 (Romanian).

[5] Andreian Cazacu, C.: Interior transformations between Klein surfaces. - To appear.

[6] BÂRZĂ, I.: Suprafețe riemanniene neorientabile. - Teză de doctorat, Bucureşti, 1980. (Sur la ramification des recouvrements riemanniens non orientable. To appear).

[7] Hurwitz, A.: Über Riemann'sche Flächen mit gegebenen Verzweigungspunkten. - Math. Ann. 39, 1891, 1-61.

[8] KeréKJáRTó, B.: Vorlesungen über Topologie. I. - Die Grundlehren der mathematischen Wissenschaften 8. Springer-Verlag, Berlin, 1923.

[9] LeHTo, O.: Riemann surfaces. - Lecture notes, University of Minnesota, Minneapolis, Minnesota, 1970.

[10] Nevanlinna, R.: Analytic functions. - Die Grundlehren der mathematischen Wissenschaften 162. Springer-Verlag, Berlin-Heidelberg-New York, 1970.

[11] Stoïlow, S.: Du nombre des points de ramification des transformations intérieures sur une variété topologique à deux dimensions. - Bull. Sci. Math. (2) 57, 1933, 355-376.

[12] Stoïlow, S.: Leçons sur les principes topologiques de la théorie des fonctions analytiques. Gauthier-Villars, Paris, 1938, and ed 1956.

University of Bucharest

Faculty of Mathematics

Bucharest 1

Romania

Received 2 April 1984 